J. Clin. Chem. Clin. Biochem.

Vol. 15, 1977, pp. 303-306

\title{
Bestimmung der Gesamtporphyrine in Urin durch Derivativ-Spektroskopie
}

\author{
Von A. Schmitt \\ Bodenseewerk Perkin-Elmer \& Co. GmbH, Überlingen
}

(Eingegangen am 14. September/13. Dezember 1976)

Zusammenfassung: Das Prinzip der Derivativ-Spektroskopie ist die Grundlage eines neuen Verfahrens zur Bestimmung der Gesamtporphyrine in Urin. Die 2. Ableitung des Normalspektrums isoliert die Soret-Bande der Porphyrine aus der Hintergrundabsorption und ermöglicht die quantitative Auswertung ohne Hilfe von Korrekturfaktoren. Das neue Verfahren ist gegenüber der klassischen Methode der Absorptionsmessung bei drei Wellenlängen um ein Mehrfaches empfindlicher und ermöglicht auch zuverlässige Bestimmungen im Normalbereich. Die kurze Analysendauer und der geringe Materialverbrauch erlauben ein wirtschaftliches Screening. Normale und leicht erhöhte Porphyringehalte werden in unverdünnten Urinproben bis zu $0,16 \mu \mathrm{mol} / 1$ sicher erfaßt, bei höheren Konzentrationen muß vorverdünnt werden. Die Empfindlichkeit für 0,01 $\mu \mathrm{mol} / 1 \mathrm{Gesamtporphyrin} \mathrm{in} \mathrm{Urin} \mathrm{ist} \mathrm{0,06} \mathrm{Absorptionseinheiten,} \mathrm{der} \mathrm{Variationskoeffizient}$ für die Serienbestimmung mit Proben von $0,07 \mu \mathrm{mol} / 1$ liegt bei $1 \%$.

\section{Determination of total urinary porphyrins by derivative spectroscopy}

Summary: The principle of derivative spectroscopy is the basis of a new procedure for determining total urinary porphyrins. The 2 nd derivative of the normal spectrum isolates the Soret band of the porphyrins from the background absorption and enables the quantitative evaluation without correction factors. The new procedure is more sensitive than the classical method using measurement at three wavelengths and permits reliable determinations in the normal range. The short analysis time and the low reagent requirement allow economic screening. Normal and slightly increased contents of total urinary porphyrins can be reliably determined in undiluted urine samples up to $0.16 \mu \mathrm{mol} / 1$. A dilution is required for higher concentrations. The sensitivity for $0.01 \mu \mathrm{mol} / 1$ total porphyrins in urine is 0.06 absorbance units. The coefficient of variation for the series determination of samples with $0.07 \mu \mathrm{mol} / 1$ is about $1 \%$.

\section{Einführung}

Die Bestimmung der Porphyrine erfolgt praktisch immer auf fluorometrischem oder spektralphotometris chem Wege. Für die Gesamtporphyrine (Uro- und Koproporphyrin) ist vorwiegend die Spektralphotometrie von Bedeutung. Grundlage dieser Bestimmung ist die intensive Soret-Bande bei $400-410 \mathrm{~nm}$. Da in diesem Bereich auch andere Bestandteile des Ürins absorbieren, muß die Interferenz dieser Stoffe eliminiert werden. Man mißt die Absorption im Maximum der Soret-Bande und bei zwei äquidistanten Wellenlängen und berechnet den Gehalt der Gesamtporphyrine mit Hilfe von Korrekturfaktoren $(1,2)$. Diese Methode liefert jedoch nur bei erhöhten Gehalten brauchbarê Wêrte (3), Normalwertsbestimmungen sind nicht durchführbar. Als Verbesserung gilt die Bestimmung nach chromatographischer Isolierung der Porphyrine über einen Anionenaustauscher mit anschließender spektrophotometrischer Bestimmung ebenfalls nach dem Prinzip der Absorptionsmessung bei drei Wellenlängen (4). Dieses Verfahren ist jedoch relativ arbeitsaufwendig und führt, insbesondere bei geringen Porphyringehalten, nicht immer zu zuverlässigen Werten.

Einen eleganten Weg für die Bestimmung der Gesamtporphyrine bietet die Ableitungs-Spektroskopie. Eine gute allgemeine Ubersicht über die Theorie und Leistung der Ableitungs-Spektroskopie wurde von $O$ 'Haver \& Green gegeben $(5,6)$. Durch dieses Prinzip erreicht man die Eliminierung der unspezifischen Hintergrundabsorption bei mehrfacher Steigerung der photometrischen Empfindlichkeit. Dabei erfordert das neue Prinzip weniger Zeitaufwand als die genannten spektrophotometrischen Verfahren, und erlaubt durch die höhere Empfindlichkeit eine zuverlässigere Bestimmung von normalen oder nur leicht erhöhten Porphyringehalten. Voraussetzung für die Durchführung der neuen 
Methode ist ein registrierendes Spektralphotometer mit einem elektronischen Differenzierzusatz, der die Aufnalume der 2. Ableitung des Spektrums im Bereich der Soret-Bande ermöglicht.

\section{Material und Methoden}

\section{Reagenzien}

Salzsäure, $320 \mathrm{~g} / \mathrm{kg}$, zur Analyse; Kontroll-Urin, pathologisch (Lederle Diagnostica, München). Frische Urinproben, bis zur Analyse lichtgeschützt aufbewahrt. Trübe Proben filtriert.

\section{Gerät}

Spektrophotometer Modell 200 oder 550 mit Schreiber und elektronischem Differenzierzusatz der Firma Bodenseewerk Perkin-Elmer \& Co., GmbH, Überlingen.

Bestimmung der Gesamtporphyrine durch DerivativSpektroskopie

Eine $1 \mathrm{~cm}$-Meßküvette enthält eine frische Mischung von $2 \mathrm{ml}$ Probe (Urin, Kontroll-Urin oder deren Verdünnung) und 0,1 $\mathrm{ml} \mathrm{HCl}(320 \mathrm{~g} / \mathrm{kg})$, die Referenzküvette enthält Wasser. Aufnahme des Spektrums in der 2. Abteilung zwischen 500 und $350 \mathrm{~nm}$. Der Differenzierungssatz ist zwischen Schreiberausgang und Schreiber geschaltet. Einstellungen am Differenżierzusatz: 2. Ableitung, Mode 6. Einstellungen am Spektrophotometer: Wellenlängenvorschub $120 \mathrm{~nm} / \mathrm{min}$, spektrale Auflösung $2 \mathrm{~nm}$, medium response, Meßbereich A 0-1.

\section{Auswertung der Derivativ-Spektren}

Für die Porphyrinbestimmung ist nur das Peakminimum bei $400 \mathrm{~nm}$ von Interesse. Quantitative Auswertung durch Entnahme der Absorptionsdifferenz zwischen Peakminimum und benachbartem Maximum bei $408 \mathrm{~nm}$. Die Absorptionsdifferenz ist der Konzentration an Gesamtporphyrinen proportional.

\section{Kalibrierung}

Die Porphyrinbestimmung durch Derivativ-Spektroskopie erfaßt die Gesamtporphyrine, die sich im Normalfall im Verhältnis 4:1 (7) auf Kopro- und Uro-Porphyrin verteilen. Da sich beide Komponenten in ihrem molaren Absorptionskoeffizienten um etwa $10 \%$ unterscheiden, ist die Kalibrierung unproblematisch. Für die Praxis hat es sich bewährt, mit einem Kontroll-Ürin zu arbeiten und die Summe der Gehalte an Kopro- und Uro-Porphyrin in $\mathrm{mmol} / \mathrm{l}$ der Kalibrierung zugrunde zu legen. Dadurch wird erreicht, daß im Normalfall der durch die Kalibrierung bedingte systematische Fehler unterhalb $5 \%$ bleibt. Die von uns verwendete Standardprobe (Lederle Abnormal Control Urin, Lot. Nr. 2921-695 R2) enthielt $0,8 \mu \mathrm{mol} / 1$ Gesamtporphyrin $(525 \mu \mathrm{g} / 1)$. Zur Kalibrierung wurde diese Probe nach 1:5-Verdünnung eingesetzt.

\section{Ergebnisse und Diskussion}

\section{Normalspektrum und 2. Ableitung}

Abbildung 1 zeigt die Normalspektren und deren 2. Ableitung eines verdünnten Kontroll-Urins (a) und einer Urinprobe (b). Das Normalspektrum des verdünnten Kontroll-Urins (1:5 mit dest. Wasser) zeigt die SoretBande der Porphyrine bei $405 \mathrm{~nm}$ bei mäßiger Hintergrundabsorption. Die 2. Ableitung isoliert diese Bande,

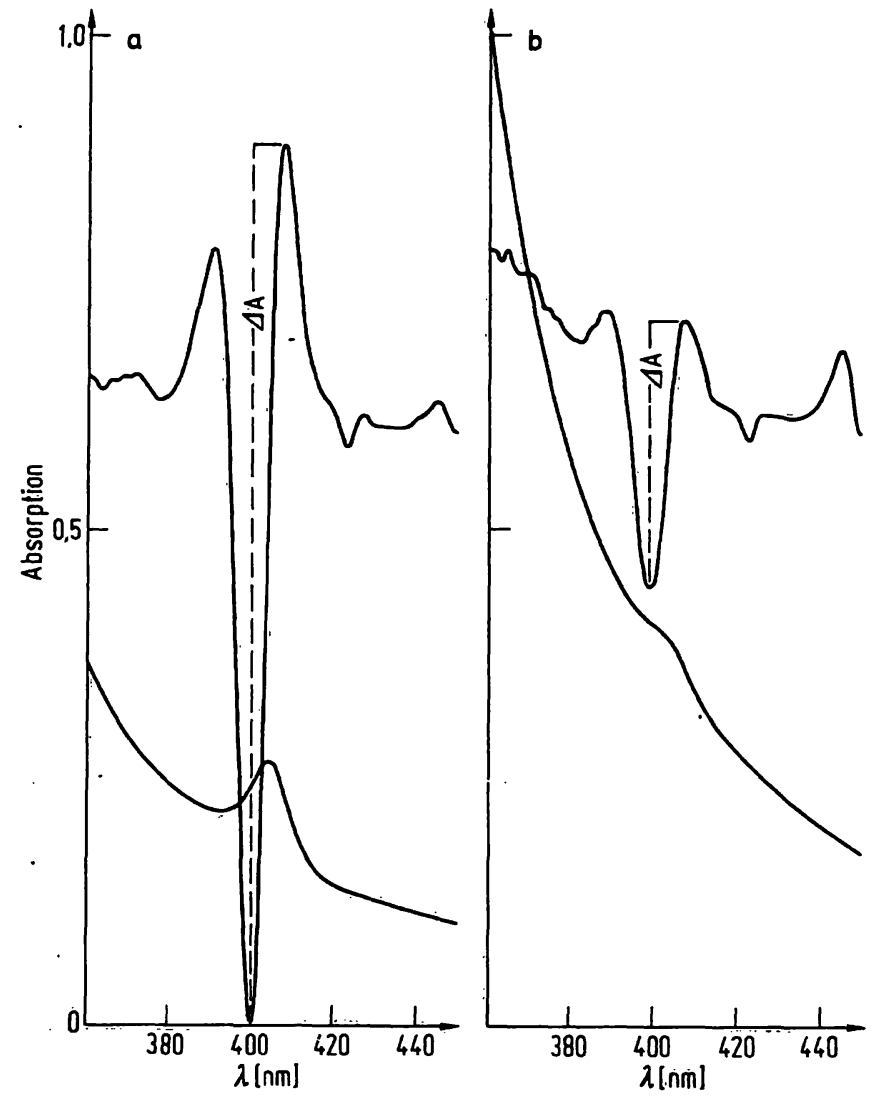

Abb. 1. Normalspektrum und dessen 2. Ableitung eines angesäuerten, verdünnten Kontroll-Urins ( $a ; 0,16 \mu \mathrm{mol} / 1$ Gesamt-Porphyrin) und einer Urinprobe (b; $0,05 \mu \mathrm{mol} / \mathrm{l}$ Gesamt-Porphyrinin).

die stetig ansteigende Hintergrundabsorption wird eliminiert und die Empfindlichkeit um ein Mehrfaches gesteigert. Bedingt durch die Differenziertechnik ist das der Soret-Bande entsprechende Peakminimum . in der 2. Ableitung um etwa $5 \mathrm{~nm}$ verschoben. Dies hat jedoch auf die quantitative Auswertung keinen Einfluß. Bei der Urinprobe mit geringerem Porphyringehalt (b) ist die Soret-Bande bei einem größeren Absorptionshintergrund nur noch als leichte Schulter angedeutet. Auch hier wird durch das 2. Ableitungsspektrum die ,verborgene" Soret-Bande aus der Hintergrundabsorption isoliert und quantitativ aușwertbar. Der Gehalt an Gesamtporphyrinen (Uro- und Koproporphyrin) ist der Peakdifferenz $\Delta \mathrm{A}$ proportional. Bezogen auf den Gehalt im verdünnten Kontroll-Urin $(0,16 \mu \mathrm{mol} / \mathrm{l})$ enthält die Urinprobe in Abbildung 10,05 $\mu \mathrm{mol} / \mathrm{l}$.

\section{Linearität und Empfindlichkeit}

Von zwei Urinproben mit erhöhten Porphyringehalten wurden Verdünnungsreihen hergestellt und nach der unter Methodik angegebenen Vorschrift das 2. Ableitungsspektrum jeder Verdünnung aufgezeichnet. Auswertung dieser Versuche in Abbildung 2: Die Peakdifferenzen $\triangle \mathrm{A}$ der Soret-Bande verhalten sich linear zum Verdünnungsgrad. Als Gesamt-Porphyrin berechnet, besteht eine lineare Beziehung bịs über $0,16 \mu \mathrm{mol} / 1$, bei họ̆heren Gehalten sind 


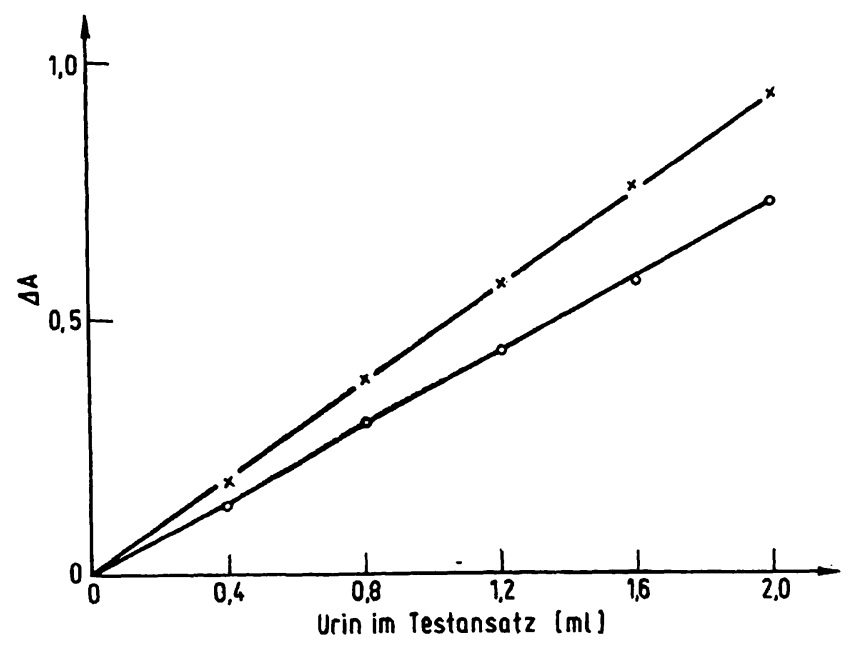

Abb. 2. Linearität der Porphyrinbestimmung durch DerivativSpektroskopie. Ergebnisse mit Verdünnungsreihen von zwei Urinen erhöhter Porphyrinkonzentration. Endvolumen des Testansatzes: $2,1 \mathrm{ml}$.

die Proben im Verhältnis 1:5 oder 1:10 mit dest. Wasser vorzuverdünnen. Die Empfindlichkeit des neuen Verfahrens kann aus Abbildung 1 entnommen werden: 0,01 $\mu \mathrm{mol} / 1 \mathrm{Gesamtporphyrin} \mathrm{in} \mathrm{Urin} \mathrm{zeigen} \mathrm{im} \mathrm{2.} \mathrm{Ablei-}$ tungsspektrum eine Peakdifferenz $\Delta \mathrm{A}$ in Höhe von 0,06 Absorptionseinheiten.

\section{Reproduzierbarkeit}

Zunächst wurde die Reproduzierbarkeit der Aufzeichnung des 2. Ableitungsspektrums mit einer Urinprobe erhöhten Porphyringehaltes ermittelt: Die nacheinander folgende Aufzeichnung des Ableitungsspektrums vom gleichen Testansatz brachte folgende Ergebnisse: $\Delta \mathrm{A}=0,855 ; \mathrm{s}=0,0044 ; \mathrm{CV}=0,5 \%$. Bei 10 in Serie separat durchgeführten Testansätzen einer Urinprobe mit etwa $0,07 \mu \mathrm{mol} / \mathrm{l}: \Delta \mathrm{A}=0,457 ; \mathrm{s}=0,0039 ; \mathrm{CV}=0,9 \%$.

\section{Zusatzversuche}

5 Urinen extrem geringer Porphyrinkonzentration wurden mit einem Kontroll-Ữrin $(0,8 \mu \mathrm{mol} / 1)$ im Verhälțis 1:5 verdünnt und wie unter Methodik angegeben analysiert. Bezogen auf den mit Wasser verdünnten Kontroll-Urin betrug die Wiederfindungsrate 90-96\%, Mittelwert $94 \%$. Die streuende negative Bilanz ist auf die unterschiedliche Hintergrundabsorption der verschiedenen Urine zurückzüü̈hren.

\section{Interferenzen}

Hohe Hintergrundabsorptionèn verursachen einen negativen Fehler, der mit steigender unspezifischer Absorption linear ansteigt. So bedingt ein Absorptionshintergrund von 0,1 Absorptionseinheiten einen negativen Fehler von etwa $1 \%$. Bei normal gefärbten Urinen bleibt der Fehler bei etwa 5\%. Bei Urinen mit erhöhtem Porphyringehalt reduziert sich dieser Fehler weitgehend, da bei solchen Proben nach Verdünnung (1:5 oder 1:10) gearbeitet werden muß.
Speziell untersucht wurde der Einfluß von Hämoglobin und Bilirubin. Zusätze von $500 \mathrm{mg} / 1$ Hämoglobin zeigten keinen signifikanten Einfluß. $20 \mathrm{mg} / 1$ Bilirubin verursachen einen negativen Fehler von etwa $10 \%$. Vor solchen Fehlern wird man jedoch gewarnt, da Bilirubinhaltige Urine im Normalspektrum bei $490 \mathrm{~nm}$ ein Maximum und im Ableitungsspektrum ein noch ausgeprägteres Minimum zeigen.

\section{Lichtempfindlichkeit}

Die Lichtempfindlichkeit der Porphyrine ist bekannt, wurde aber bisher nicht systematisch untersucht. In eigenen Versuchen wurde der Porphyringehalt zweier Urinproben durch Zugabe von pathologischem KontrollUrin aufgestockt und die Lichtempfindlichkeit nach der Methode der Ableitungsspektroskopie über mehrere Stunden verfolgt. Die Proben (pH-Werte 5,6 und 5,8) wurden der normalen Tagesbeleuchtung im Labor in kleinen Bechergläsern ausgesetzt, Kontrollen wurden lichtgeschützt in dunklen Flaschen aufbewahrt. Ein mit $\mathrm{HCl}$ angesäuerter Testansatz wurde ebenfalls belichtet und in gleichen Zeitabständen analysiert. Die Ergebnisse dieser Versuchsreihe sind in Abbildung 3 zusammengestellt. Während die lichtgeschützte Urinprobe (I) auch nach drei Stunden keine Änderung des Porphyringehaltes zeigt, nimmt in den belichteten Proben (II, III) der Gehalt sehr schnell ab: Nach drei Stunden Belichtung wurden nur noch etwa $30 \%$ des ursprünglichen Porphyringehaltes gefunden. In Urinproben, die wie unter Methodik angegeben, mit Salzsäure angesäuert wurden, bleibt der Porphyringehalt auch bei mehrstündiger Belichtung konstant. Eine mit der Zeit zunehmende Rotfärbung der angesäuerten Proben ist ohne Einfluß auf den ermittelten Porphyringehalt. Urinproben, die nicht bald zur Analyse gelangen, sollten daher lichtgeschützt aufbewahrt oder angesäuert werden.

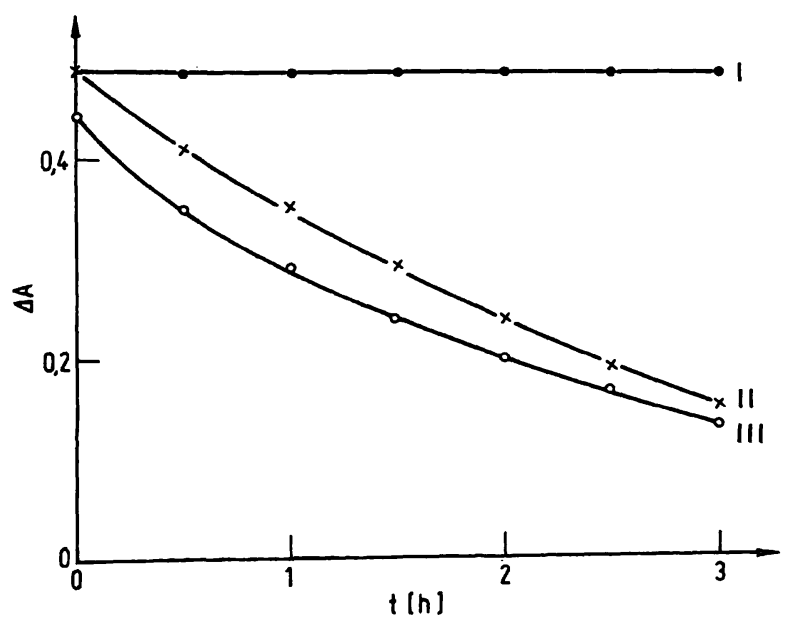

Abb. 3. Lichtempfindlichkeit der Porphyrine in Urin. - Urinprobe in dunkler Flasche. $x-x$ und $0-0$ Urinproben im Becherglas bei normaler Tagesbeleuchtung im Labor. 


\section{Literaturverzeichnis}

1. Rimington, C. \& Sveinsson, S. L. (1950), Scand. J. Clin. Lab. Invest. 2, 209-216.

2. Schlenker, F. S. \& Kitchel, C. L. (1958), Am. J. Clin. Pathol. 29, 593-597.

3. Richterich, R. (1971), Klinische Chemie, Theorie und Praxis, 3. Auflage, Karger-Verlag, Basel, 417-423.

4. Doss, M. \& Schmidt, A. (1971), diese Z. 9, 415-418.

5. O’Haver, T. C. \& Green, G. L. (1975), Intern. Lab. 5/6, $11-17$.

6. O'Haver, T. C. \& Green, G. L. (1976), Anal. Chem. 48, 312 $-318$

7. Doss, M. \& Meinhof, W. (1971), Dtsch. Med. Wochenschr. 96, 1006-1013.

Dr. Albert Schmitt

Bodenseewerk Perkin-Elmer \& Co. GmbH

Postfach 1120

D-7770 Überlingen 